\title{
Phase Space Topography and the Lyapunov Exponent of Electrocorticograms in Partial Seizures
}

\author{
Leonidas D. Iasemidis", J. Chris Sackellares", Hitten P. Zaveri", and William J. Williams*
}

\begin{abstract}
Summary: Electrocorticograms (ECoG's) from 16 of 68 chronically implanted subdural electrodes, placed over the right temporal cortex in a patient with a right medial temporal focus, were analyzed using methods from nonlinear dynamics. A time series provides information about a large number of pertinent variables, which may be used to explore and characterize the system's dynamics. These variables and their evolution in time produce the phase portrait of the system. The phase spaces for each of 16 electrodes were constructed and from these the largest average Lyapunov exponents (L's), measures of chaoticity of the system (the larger the L, the more chaotic the system is), were estimated over time for every electrode before, in and after the epileptic seizure for three seizures of the same patient. The start of the seizure corresponds to a simultaneous drop in $L$ values obtained at the electrodes nearest the focus. $L$ values for the rest of the electrodes follow. The mean values of $L$ for all electrodes in the postictal state are larger than the ones in the preictal state, denoting a more chaotic state postictally. The lowest values of L occur during the seizure but they are still positive denoting the presence of a chaotic attractor. Based on the procedure for the estimation of $\mathrm{L}$ we were able to develop a methodology for detecting prominent spikes in the ECoG. These measures ( $\left.\mathrm{L}^{*}\right)$ calculated over a period of time (10 minutes before to 10 minutes after the seizure outburst) revealed a remarkable coherence of the abrupt transient drops of $L^{*}$ for the electrodes that showed the inital ictal onset. The $L^{*}$ values for the electrodes away from the focus exhibited less abrupt transient drops. These results indicate that the largest average Lyapunov exponent $\mathrm{L}$ can be useful in seizure detection as well as a discriminatory factor for focus localization in multielectrode analysis.
\end{abstract}

Key words: phase space; chaos; Lyapunov exponents; ECoG; partial epileptic seizures; epileptogenic focus localization.

\section{Introduction}

Long-term recordings of brain electrical activity recorded from scalp and sphenoidal electrodes, depth electrodes or subdural electrodes are employed in our clinical laboratories to localize the origin of seizure discharges in patients with partial (focal) seizures who are candidates for surgical removal of the seizure focus. Currently, in clinical practice, the recordings are analyzed visually to identify and localize interictal and ictal epileptogenic discharges. Computerized systems may be employed to detect epochs of the signal that may contain interictal spikes or ictal discharges. The epochs of interest are then subjected to visual interpretation. Most computerized systems are designed to detect signal transients

*Bioengineering Program Department of Electrical Engineering and Computer Science.

**Epilepsy Program Department of Neurology University of Michigan, Ann Arbor, Michigan.

(Accepted for publication: January 12, 1990).

Correspondence and reprint requests should be addressed to J. Chris Sackellares, M.D., Epilepsy Program, Department of Neurology, 1920 Taubman Center/0316, 1500 E. Medical Center Drive, Ann Arbor, MI, USA 48109-0316.

Copyright (01990 Human Sciences Press, Inc. such as spikes, spike and slow wave complexes or rhythmic slow wave bursts that meet certain waveform criteria such as frequency, amplitude and morphology (Gotman et al. 1985). Recently, we have approached the problem of computer-assisted localization of the seizure focus in electrocorticograms using phase-space topography.

In this approach, the voltages of the recorded signal $x(t)$ at several points in time $[t, t+\tau, \ldots, t+(p-l) \cdot \tau]$, separated by a given time lag $\tau$, are converted to a $p$ dimensional vector $X(t)$. The process is repeated by increasing $t$ to give successive points in the phase space, thus creating a $\mathrm{p}$-dimensional structure. This portrait can provide a visual image of the behavior of the signal over time (especially when $\mathrm{p}=2$ or 3 ). The phase space portrait can be analyzed mathematically to demonstrate the presence of an attractor (region of the phase space of lower dimension, within which the signal is confined in its future evolution) and its dimension. The presence of an attractor, for a particular time period, of a low dimension means that of the many (possibly infinite) modes of the system only a portion of them are active during this period. Then the effective phase space dimension is much smaller than the full dimension of the equations describing the system. This assumes a change either in the parameters (autonomous system - structural change) 


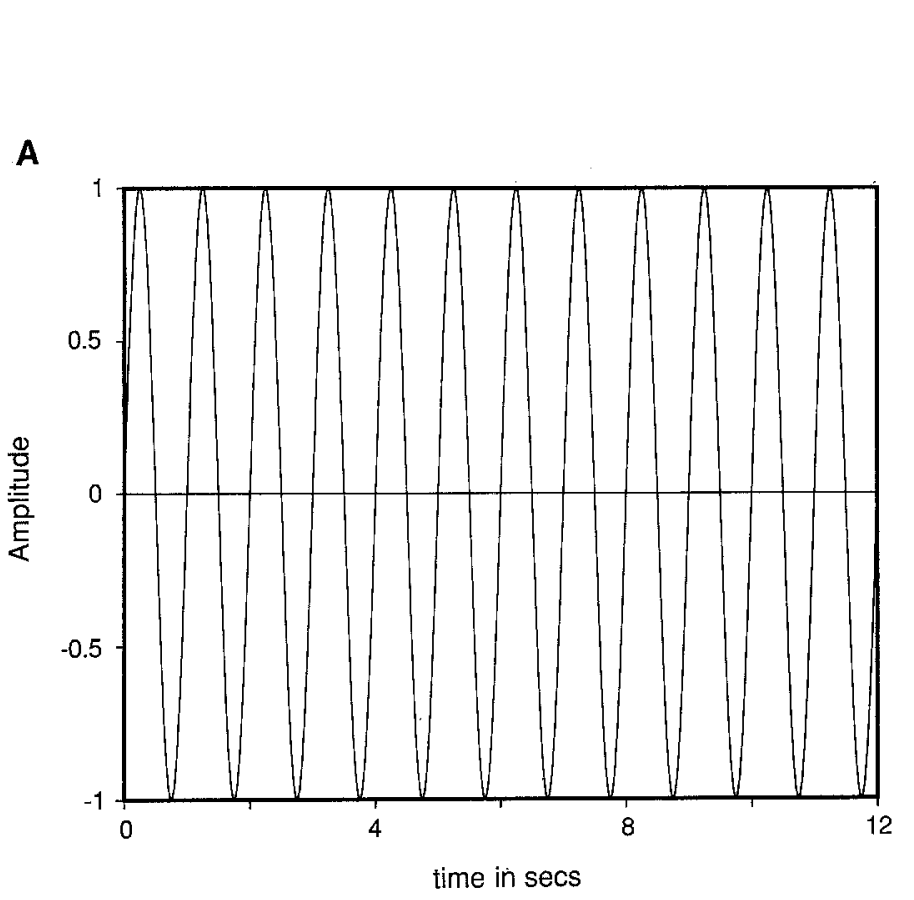

B

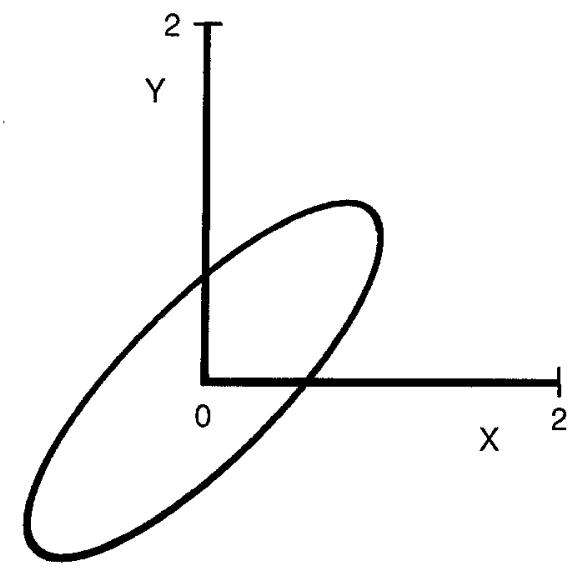

C

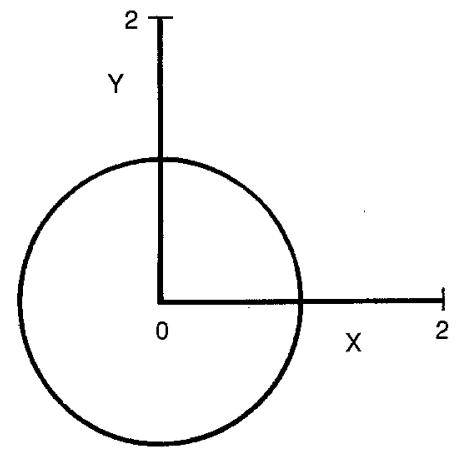

Figure 1 (a) Computer generated $1 \mathrm{~Hz}$ sine wave in time domain. (b) Phase space plot (projection on the $X-Y$ plane: $P=3$, $\tau=100 \mathrm{msec}$ ). (c) As in (b) with $\tau=250 \mathrm{msec}$.

or in its inputs, too (nonautonomous system).

Some physiologic systems have been found to behave in a non-linear chaotic fashion (Glass et al. 1988). Non-linear systems exhibit certain mathematical characteristics such as strong dependence on initial conditions and the presence of attractors of fractal dimension (strange attractors) (Ott 1981; Swinney 1983). The strong dependence on initial conditions is reflected in the phase space portrait by the rapid divergence of nearby trajectories. This rapid divergence can be expressed mathematically in terms of the average largest Lyapunov exponent L. For systems in which nearby trajectories in the phase space diverge exponentially, $\mathrm{L}$ is positive (for chaotic systems at least one positive Lyapunov exponent is present). When trajectories are parallel, $\mathrm{L}$ is 0 . Converging trajectories yield negative values for L. (For concepts and definitions see Appen$\operatorname{dix} A$ ).

Methods have been developed to determine the presence of an attractor, its dimension (Holzfus et al. 1986 and Layne et al. 1986) and values for the Lyapunov exponent. Babloyantz and co-workers have applied similar methods to show the existence of an attractor in an instance of petit mal epilepsy (Babloyantz et al. 1986) and at different stages of the sleep cycle in normal persons (Babloyantz et al. 1985). They demonstrated the presence of attractors of low and fractal dimension and positive values for $\mathrm{L}$ within the attractor. All of these findings are consistent with the behavior of a non-linear chaotic system (Mayer-Kress et al. 1987).

Our initial findings in the ECoG of partial (focal) epilepsy of temporal lobe origin indicate that the epileptogenic focus also generates signals characteristic of a non-linear dynamic system. (Iasemidis et al. 1988a). Analysis of signals from a single subdural electrode near the center of the epileptogenic focus demonstrated strange attractors of low dimension and positive values for $\mathrm{L}$ during the immediate preictal period, during the seizure (ictal), and following the seizure (postictal); (Iasemidis et al. 1988b). We found different phase portraits for each stage of the seizure. In addition, as the signal evolves from preictal to ictal to postictal phases, there are characteristic changes in the values for the Lyapunov exponent. These findings suggested that phase space analysis might provide a means for detecting and localizing the onset of a seizure. In the following examples, the application of phase space topography to signal analysis will be illustrated. 


\section{Phase Space Topography of Sine Waves}

The phase space portrait of a signal provides a visual image of the evolution over time. Its characteristics reflect the characteristics of the original signal. The theoretical basis for this relationship has been given by Packard and coworkers (1980) and Takens (1981) and developed from the Whitney embedding theorem (Whitney 1936).

An intuitive understanding of how the original signal characteristics are reflected in the phase space can be gained by constructing phase space portraits of elementary signals. For example, a $1 \mathrm{~Hz}(\mathrm{~T}=1 \mathrm{sec})$ sine wave is shown in Figure 1a. The phase space plot was constructed with $p=3$ and a time lag $\tau=0.1 \mathrm{sec}$, which corresponds to a phase difference $\varphi=\left(2 \pi^{*} 0.1\right)$ radians between the components of each vector of the phase space. The resulting curve is an ellipse whose shape depends on $\varphi$. The projection of the phase space on the $X-Y$ plane is shown in Figure $1 \mathrm{~b}$. By changing the value

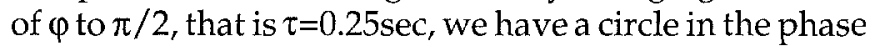
space (Figure 1c).

Figure 2a shows a signal generated by the addition of

A

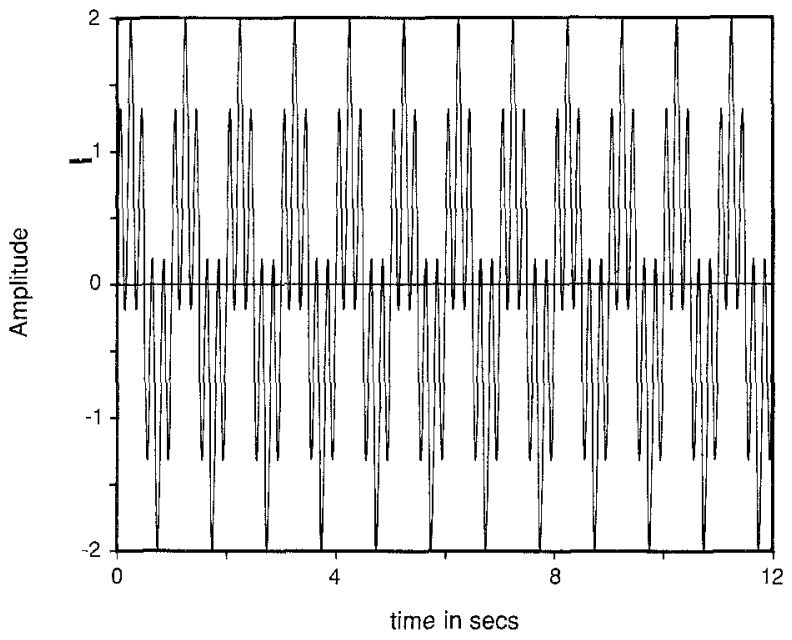

$1 \mathrm{~Hz}$ and $5 \mathrm{~Hz}$ equal amplitude sine waves (the ratio of the frequencies is a rational real number), which is a periodic signal with the largest period $\mathrm{T}=1 \mathrm{sec}$. The resultant phase space plot is a closed curve, since the time series is periodic, but not elliptic because the sine waves are of different frequencies (one dimensional attractor; not strange). The phase space is constructed with $\mathrm{p}=3$ and $\tau=10 \mathrm{msec}$ (Figure $2 b$ ). The phase space with $p=3$, $\tau=100 \mathrm{msec}$ is shown in Figure $2 \mathrm{c}$. Comparison of Figure $2 c$ with Figure $2 b$ illustrates the effects of the time lag $\tau$ on the construction of the phase space plot (in Figure 2c we have (p-1) ' $\tau=200 \mathrm{msec}=$ period of the $5 \mathrm{~Hz}$ sine wave; the $5 \mathrm{~Hz}$ sine wave now is seen clearly by counting the "closed" curves; the $1 \mathrm{~Hz}$ sine wave is represented by the surrounding closed loop). Figure 3 a shows a signal composed of the same $1 \mathrm{~Hz}$ and $5 \mathrm{~Hz}$ sine waves but with the two frequency components 90 degrees out of phase. Comparison of Figure $3 b$ with Figure $2 b$ shows the effect on the phase space plot of the phase delay between different components present in the signal. All of the above composed signals are periodic. Since they are periodic, irrespective of their complexity, they have a topological dimension $D=1$. In the phase space this results in a complex but closed curve (a trajectory that
B

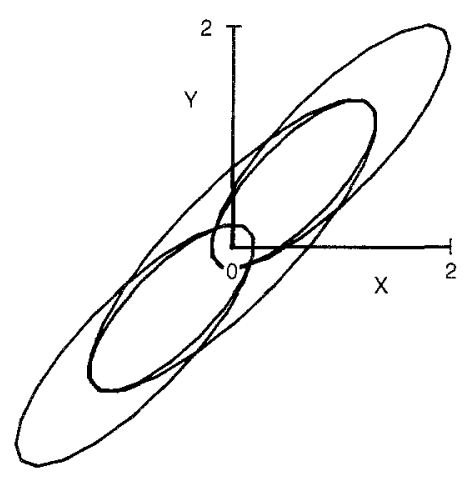

C

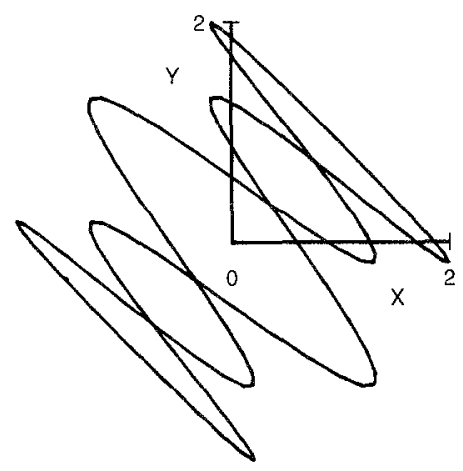

Figure 2 Computer generated signal composed of the sum of $1 \mathrm{~Hz}$ and $5 \mathrm{~Hz}$ in phase sine waves (a) in time domain. (b) Phase space plot (projection on the $X-Y$ plane; $P=3, \tau=10 \mathrm{msec}$ ). (c) As in (b) but with $\tau=100 \mathrm{msec}$. 
A

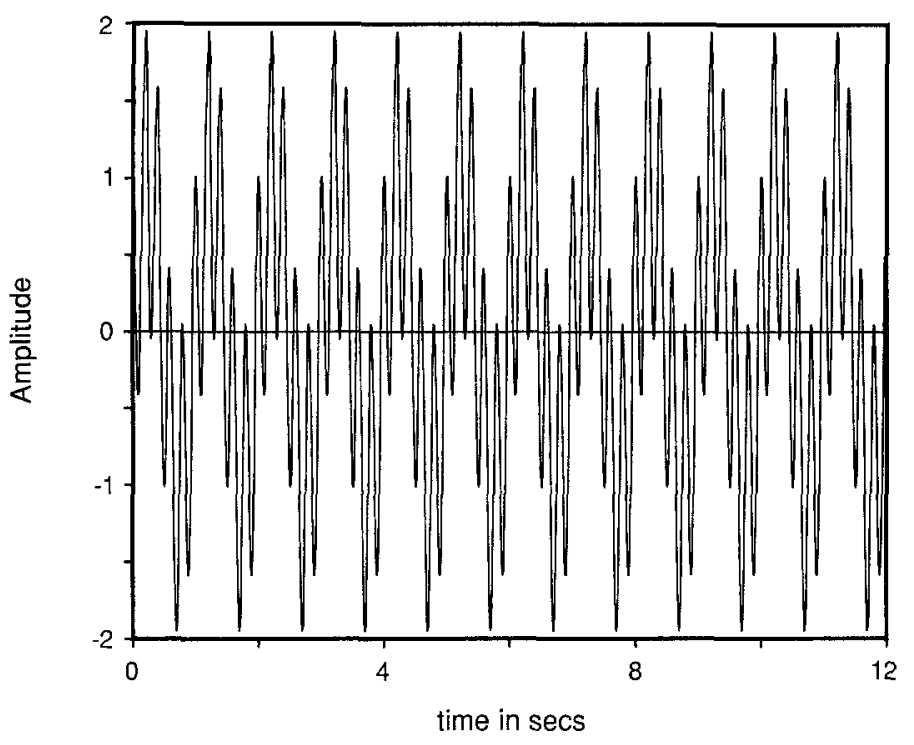

B

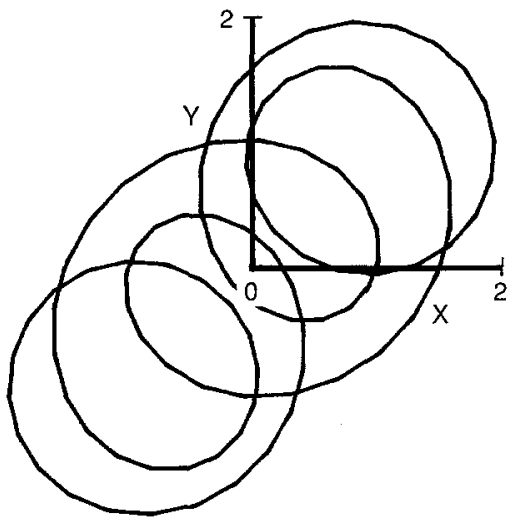

Figure 3 (a) The compound sine wave signal depicted in figure $2 a$ with the two frequency components 90 degrees out of phase. (b) Phase space plot-projection on the X-Y plane $(p=3, \tau=10 \mathrm{msec})$.

does not fill space).

In Figure 4a the signal is composed of two sines, one with frequency $1 \mathrm{~Hz}$ and the other with frequency $\sqrt{2} \mathrm{~Hz}$. The ratio of the frequencies $\sqrt{2}$ is a real irrational number and the signal is aperiodic (quasiperiodic). In the phase space it fills a surface (a 2 dimensional attractor, $D=2$; not strange). The phase space plot of this signal is a torus. One can imagine the situation by considering the two sine waves as two independent circular motions (closed curves in the phase space) transverse to each other at each of their intersection points. The composed motion will result in a complete filling of a doughnut shape surface (moving along the longitudinal direction with the low frequency sine wave and along the transverse direction with the high frequency one). In Figure $4 b$ the phase space is constructed with $p=3, \tau=10 \mathrm{msec}$ and an orthogonal projection on the $X-Y$ plane of 12 sec of the data is given. In Figure $4 \mathrm{c}$ a perspective projection onto the $X-Y$ plane is illustrated for $50 \mathrm{sec}$ of the data (the view point is on the $\mathrm{Z}$ axis). Adjacent orbits on the torus remain near one another on the long-term and predictability is guaranteed (not chaotic attractor).

\section{Strange and Chaotic Attractors in the ECOG}

The presence of an attractor can be confirmed mathematically (Grassberger et al. 1983 and Rapp et al. 1986). Since the dimension of the attractor observed in the ictal ECoG signal is low and non-integer (thin fractal) (Iasemidis et al. 1988b), it is a strange attractor. Such strange attractors are characteristic of deterministic nonlinear dissipative systems (Campbell, 1987). To determine whether the attractor is behaving chaotically, without knowing the exact equations of the system under consideration, one can compute the value of the largest Lyapunov exponent (Wolf et al. 1986). In our analysis of the ECoG the Lyapunov exponent $\mathrm{L}$ was computed by a modified version of the program proposed by Wolf et al. 1985 (see Appendix A for definitions and Appendix B for methodology and details on the modifications we introduced because of the nature of the transients inherent in the epileptic data). The other method of estimation of $\mathrm{L}$ is the one proposed by Eckmann et al. (1985) and permits an approximation of all L's. Vastano et al. (1986) compared both of the above methods and showed that the latter yields $L$ estimates that vary considerably with the embedding dimension $p$ while the former is more robust for the estimation of the largest $\mathrm{L}$. Since it is difficult to know the exact value of $p$ and its changes, especially over a period of 20 minutes, we decided to use the former method for a consistent estimation of $\mathrm{L}$. ( $\mathrm{L}$ values of known signals, for comparison reasons, are given in Appendix C; see also Figure 14).

In Figure 5a a 6 second segment of ECoG is illustrated. This ictal signal was recorded 15 seconds after the onset of a seizure from an electrode located over a right temporal lobe epileptogenic focus in a patient with medically refractory partial seizures. The sampling frequency is $500 \mathrm{~Hz}$ (sampling period $\mathrm{Dt}=2 \mathrm{msec}$ ), the maximum prominent frequency present is less than $25 \mathrm{~Hz}$, and the digital filter cut off is $50 \mathrm{~Hz}$. [The optimal low pass (symmetric impulse response) filter used was a linear phase 100 points FIR using the weighted 100:1 Chebyshev approximation-Remez algorithm]. From this ECoG signal, the phase space plot was constructed. The time lag used is $\tau=14 \mathrm{msec}$ (Appendix B-Step 2). Orthogonal projections on $X-Y$ and $X-Z$ planes are given in Figure $5 b$. For the attractor illustrated in Figure $5 b$, the largest Lyapunov exponent $\mathrm{L}$ is $0.98 \mathrm{bits} / \mathrm{sec}$. Since $\mathrm{L}$ is positive the attractor is chaotic.

Figure 6a shows a 6 second segment recorded 108 
A

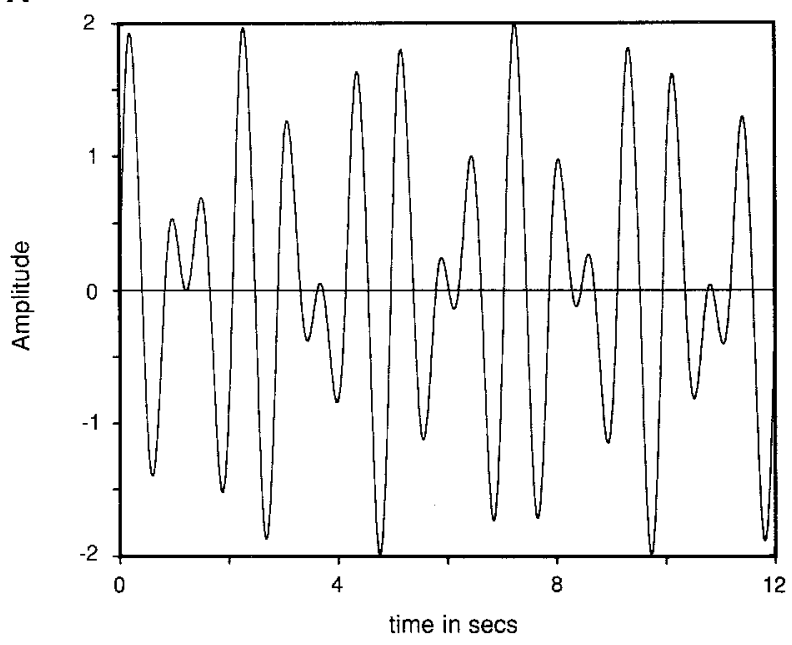

B

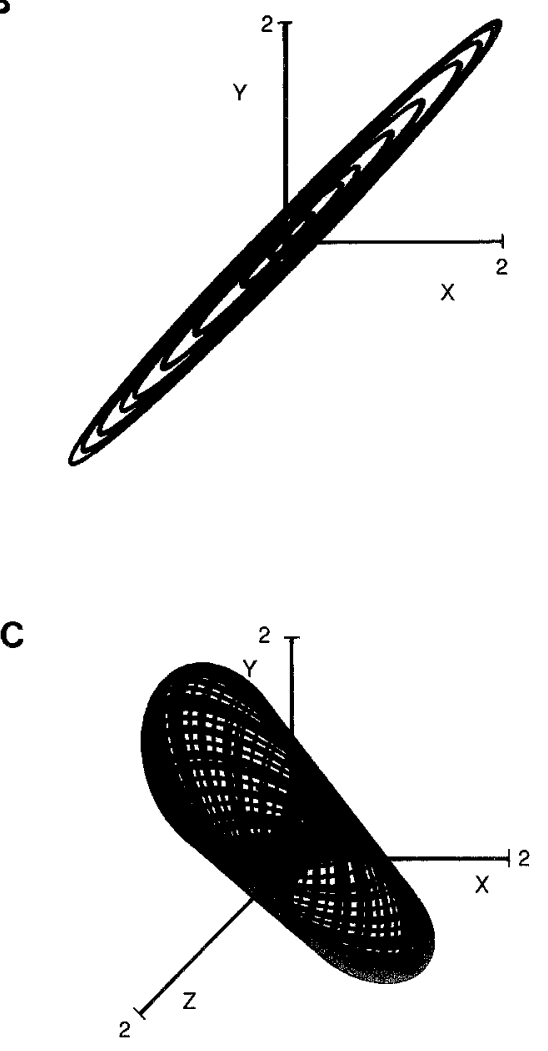

Figure 4 (a) Computer generated signal composed of the sum of $1 \mathrm{~Hz}$ and $\sqrt{2} \mathrm{~Hz}$ in phase sine waves, in time domain. (b) Phase space plot (torus) with $p=3, \tau=10$ msec (projection on $X-Y$ plane). (c) Phase space plot, with $p=3, \tau=500$ msec (perspective projection).

seconds before the onset of the seizure. Figure $6 \mathrm{~b}$ illustrates the phase space construction of the preictal signal in Figure 6a. Visual inspection of Figure $6 \mathrm{~b}$ reveals that the signal tends to be attracted to a particular region of the phase space (the one with the highest point density). The largest Lyapunov exponent is 7.56 bits/sec. Thus, the preictal signal is more chaotic than the ictal one.

Figure $7 \mathrm{a}$ is a 6 second segment recorded 15 seconds after the end of the seizure. Figure $7 \mathrm{~b}$ is its phase space plot.

Since the phase portraits observed in the preictal and ictal signals are quite different, it is interesting to follow the change in chaoticity during the transition from the preictal to the ictal state by calculating the value of the Lyapunov exponent as a function of time. Figure 8 illustrates the change in the value of the Lyapunov exponent over a period of 20 minutes, beginning 10 minutes before the seizure, continuing through the seizure to the postictal state. This plot shows that prior to the seizure, the value of $\mathrm{L}$ is high. However, there are several abrupt drops in the value of $L$ during the preictal period. At the onset of the seizure, there is a sudden drop in $L$, but as the seizure progresses, L starts increasing. In the postictal state, $\mathrm{L}$ returns to an average level higher than its preictal one. Analysis of two other seizures of the same patient revealed the same behavior. From Figure 8 it is clear that the plot of $L$ versus time provides a means for detecting the seizure discharge.

These observations suggest that monitoring the Lyapunov exponent may be useful in automatic seizure detection. The effect on $L$ of artifacts of smaller duration than the duration of the segment used to estimate $L$ (here $12 \mathrm{sec}$ ), is small. However, the effect of longer duration artifacts could be severe. Thus, the utility of this technique for monitoring scalp recorded EEG signals is yet to be determined.

\section{Multielectrode Analysis of the Preictal ECOG}

We hypothesized that analysis of the ECoG using phase space functions from multiple electrodes would distinguish between electrodes overlying interictal spike 

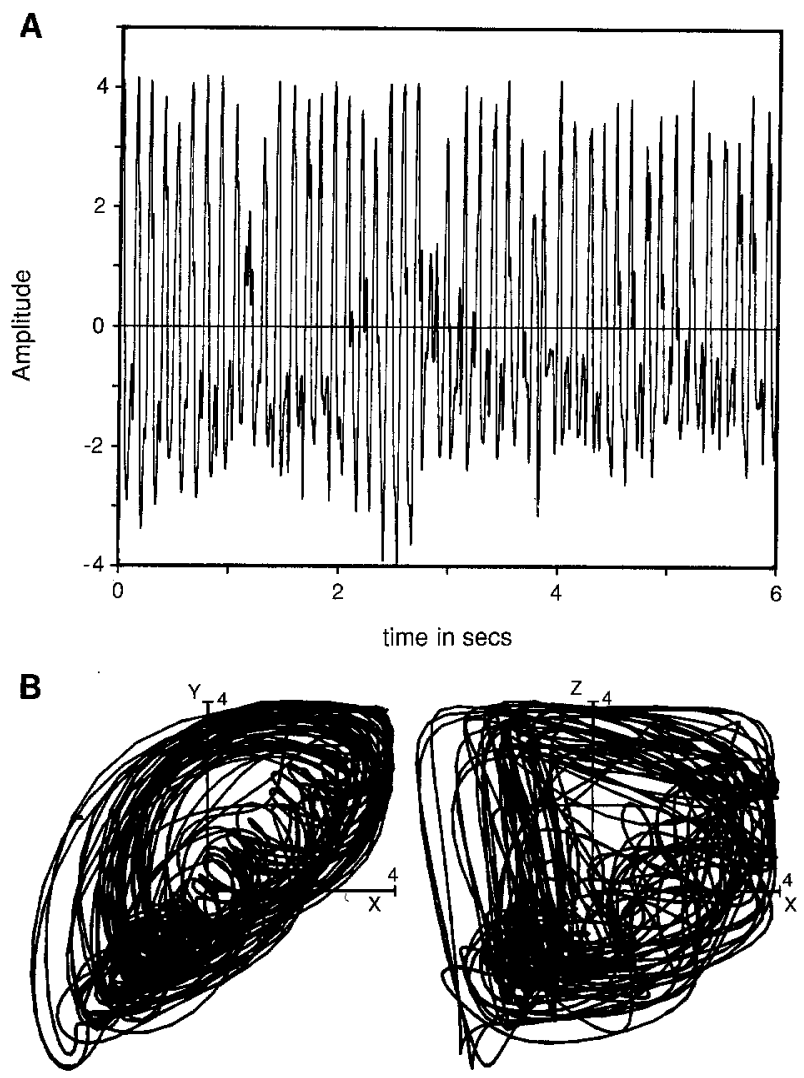

Figure 5 (a) lctal ECOG signal from electrode A3 overlying a spike focus (15 sec after the seizure onset) . (b) Orthogonal projections of the phase space of the ictal discharge depicted in (a) with $p=7, \tau=14 \mathrm{msec}$.

foci and provide a means for localizing the seizure onset. To test these hypotheses, we analyzed signals of several electrodes from the subdural array. The schematic diagram of the 68 electrodes employed in our recording is shown in Figure 9. Visual analysis of the ECoG beginning 10 minutes prior to each seizure revealed two interictal spike foci. One focus involved electrodes I1 and I2.

The second focus involved electrodes $\mathrm{Bl}, \mathrm{A} 1$ and $\mathrm{A} 3$. Figure 10 is a plot of the Lyapunov exponent versus time calculated from phase space plots of signals from electrodes B1, A1 and A3. This plot represents sequential values obtained from 12 second non-overlapping epochs for a period of time beginning 100 seconds prior to a seizure until only 80 seconds after the seizure onset. (This seizure is different from the one analyzed to obtain $\mathrm{L}$ in Figure 8; only the transition period is shown for enhancement of details).

During the preictal period, signals from each electrode exhibit positive values of $L$ with multiple transient drops. At the onset of the seizure, signals from all three electrodes exhibit a simultaneous drop in L. Visual inspection of the ECoG tracing shows that the earliest detectable ictal discharge occurs in these three electrodes. It is also interesting to note that in this seizure the mini-
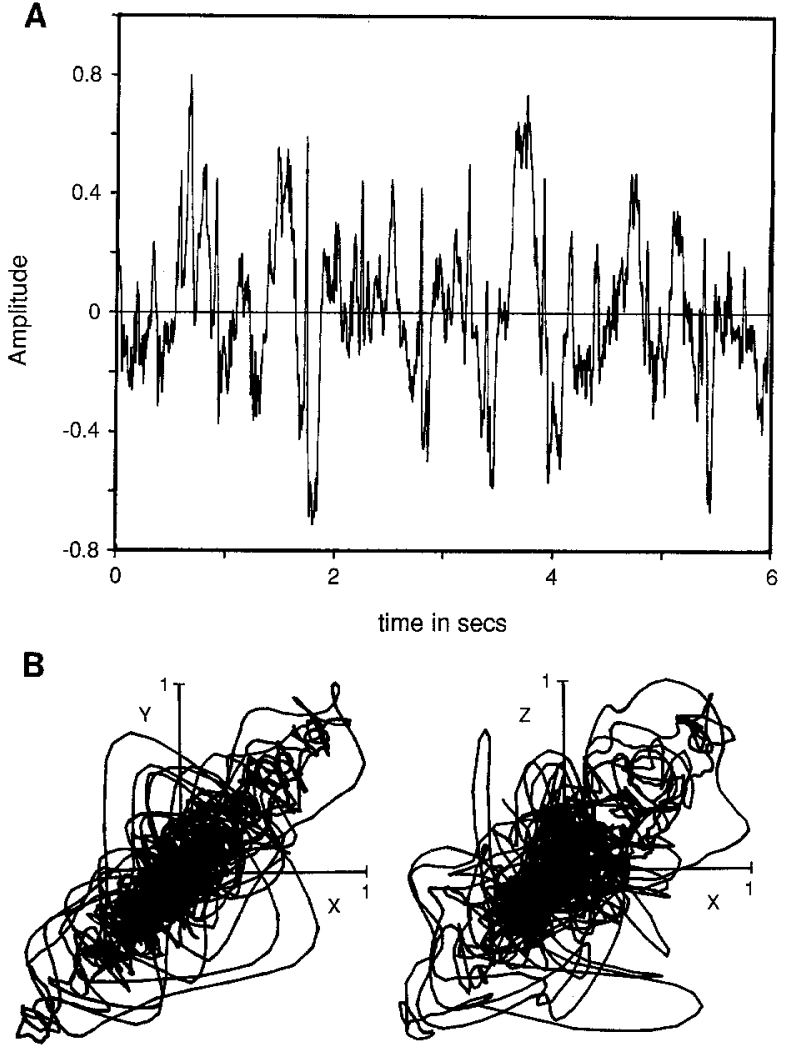

Figure 6 (a) Preictal ECOG signal from electrode A3 overlying a seizure focus ( $108 \mathrm{sec}$ before the seizure onset). (b) Orthogonal projections of the phase space of the preictal ECOG signal shown in (a) with $p=7, \tau=14 \mathrm{msec}$.

mum value of $\mathrm{L}$ for electrode $\mathrm{B} 1$ is slightly negative (at the seizure's onset). In contrast, $\mathrm{L}$ versus time (L(t)) plots for electrodes outside the focus, (electrodes D1,D3 in Figure 10), show an abrupt drop in $L$ later than that observed in the electrodes overlying the ictal focus. These findings were replicated for each of the three focal seizures analyzed in this patient.

In order to capture the most prominent spikes that occur in each segment we estimated values of $L$ modifying the range of $t_{1}$ over the duration $T$ of the whole segment under consideration (thus equating the bound IMAX of $t_{1}$ in formula (B2) of Appendix B to T). Then $X_{\max }$ corresponds to the largest amplitude spike present in the segment. The behavior of $\mathrm{L}(\mathrm{t})$ now changes dramatically under this modified analysis, emphasizing the presence of prominent spikes and deemphasizing details about the structure of the underlying process. This modified form of $\mathrm{L}(\mathrm{t})$ turns out to be very useful. We denote this measure $L^{*}(t)$. To illustrate this point, in Figure 11 we plot the $L^{*}(t)$ for electrode pairs $B 1$, $A 1$, and $B 1, D 1$. Low values of $L^{*}$ correspond to the presence of prominent spikes in the data segments. Detection of the seizure using $L^{*}$ is not possible.

Two observations are of note here. First, from Figure 

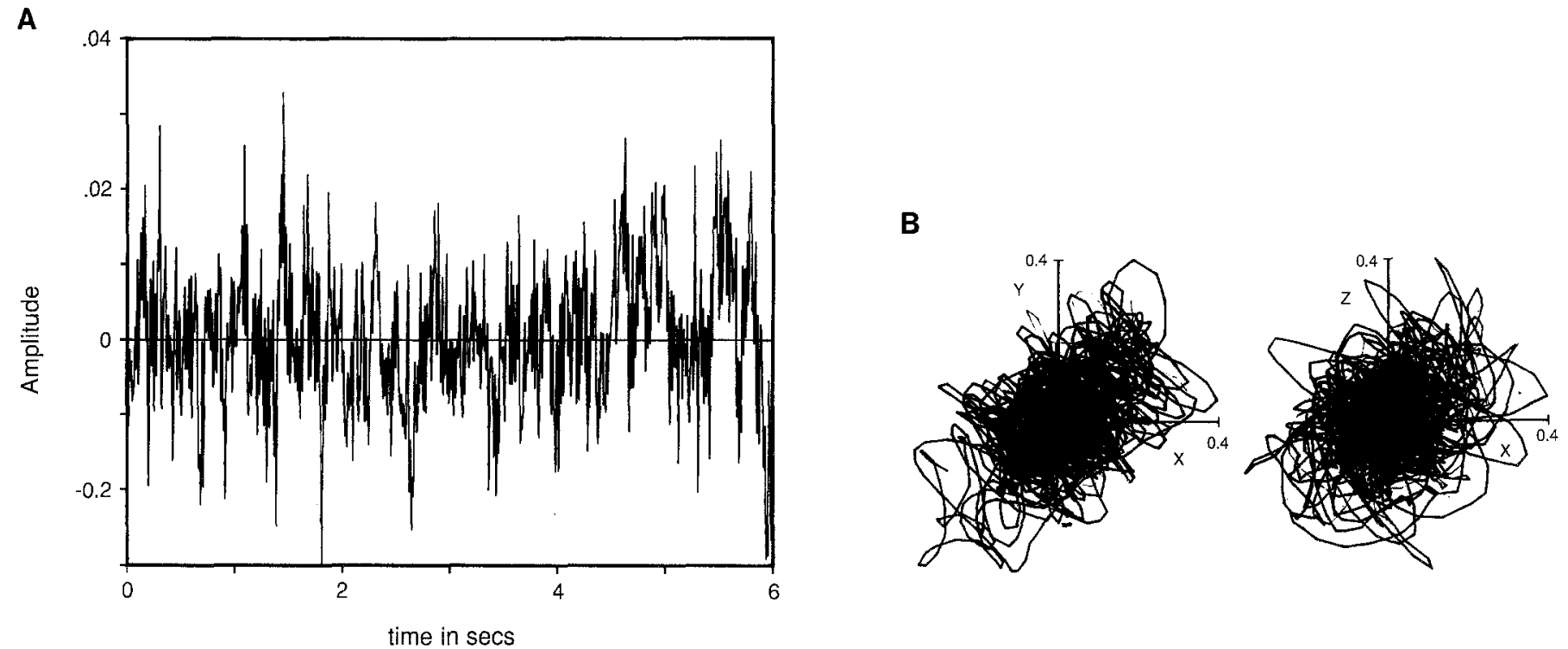

Figure 7 (a) Postictal ECOG signal from electrode A3 overlying a seizure focus ( 15 sec after the end of the seizure) (b) Orthogonal projections of the phase space of the postictal ECoG signal shown in Figure $5 a(p=7, \tau=14 \mathrm{msec})$.

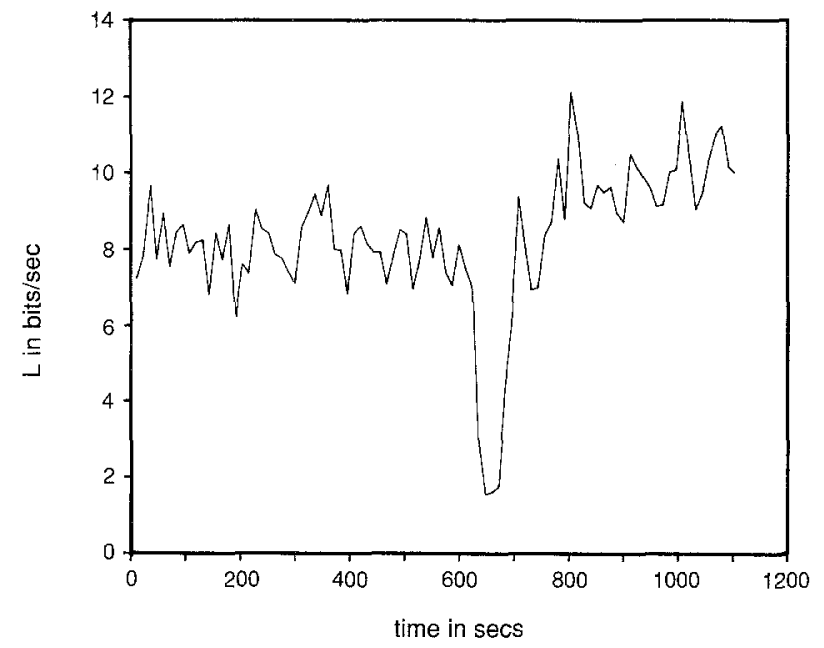

Figure 8 Plot of the Lyapunov exponent $\mathrm{L}$ over time of a signal derived from electrode $\mathrm{Al}$ overlying the focus. The value of $L$ is positive. At the onset of the seizure, $L$ drops abruptly to its lowest value. Transition to the postictal state is associated with a gradual increase in $L(p=7, \tau=14 \mathrm{msec}$, $\mathrm{T}=12 \mathrm{sec}, \Delta t=100 \mathrm{msec}, \partial_{\text {init }}=0.2 \mathrm{rad}, \operatorname{IMAX}=84 \mathrm{msec} b=0.05$, $\mathrm{c}=0.1$ ) 11a we notice many drops of $L^{*}$ and an almost complete alignment of Elec. B1 and Elec. A1, both close to the focus, with respect to the drops of $L^{*}$. Secondly, from Figure $11 \mathrm{~b}$ we notice a small number of drops in the values of $L^{*}$ estimated from Elec. D1, which is away from the focus, as well as a substantial difference in the values of $\mathrm{L}^{*}$ in the preictal state from the ones estimated from Elec. B1. This pattern was present for all seizures analyzed among the electrodes over and far away from the focus. This finding, even 10 minutes before the actual outburst, suggests a close relationship between the background spiking process and the recruitment of cortical regions during the unfolding of a seizure.

\section{Discussion}

Based on our inital studies of the ECoG in patients with partial seizures, we have found that construction of phase space plots from the original signal provides a useful method for visual and mathematical analysis of the signal. This approach to signal analysis is particularly useful for analyzing non-linear chaotic signals since it does not require complete understanding of the system producing the signal. Nor does this approach require 


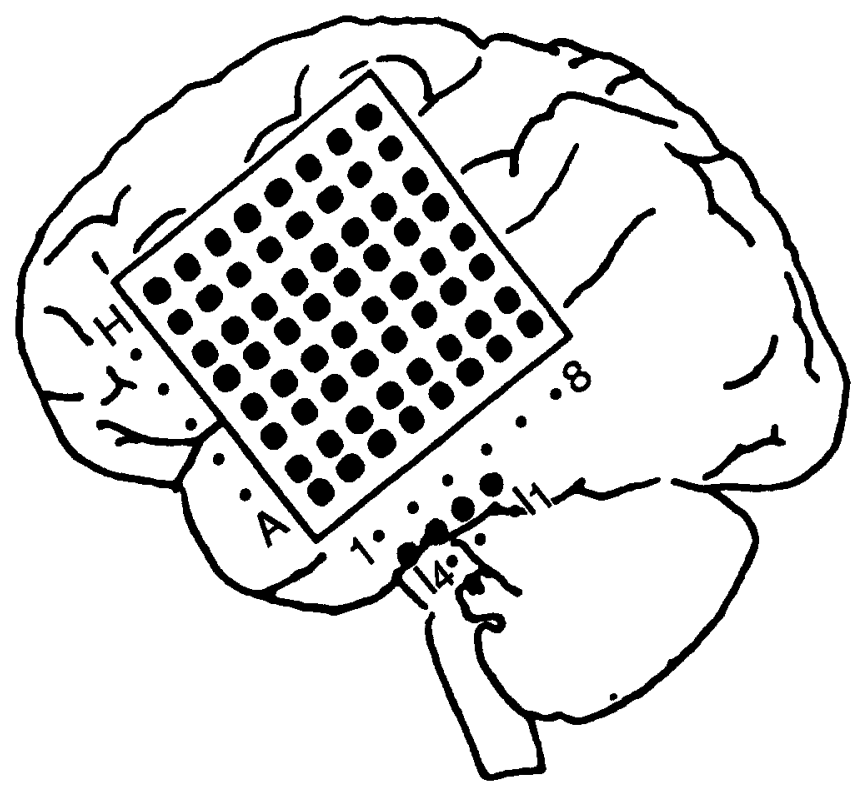

Figure 9 Schematic diagram showing the placement of the subdural electrodes used for recording the ECOG.

mathematical modelling of the system, although it may provide essential information required to develop such a model.

Our studies of the ECoG recorded in human temporal lobe epilepsy indicate that the behavior of the ECoG signal over time is characteristic of a non-linear chaotic system (Iasemidis et al. 1988a). The preictal signal is less chaotic than the postictal one. The onset of the seizure is characterized by, at least in the cases studied, simultaneous transitions to a more ordered state in signals derived from electrodes over the focus. Subsequent spread of the seizure discharge to cortical regions outside the focus is associated with similar transitions toward a more ordered state as well.

In addition to potential for practical application of these methods of seizure detection and localization, our initial findings are of theoretical interest. A number of non-linear mathematical models have been shown to exhibit chaotic behavior for certain values of the model parameters. Such mathematical models include models for excitatory feedback systems, inhibitory feedback systems and mixed (excitatory and inhibitory) feedback systems. Of particular interest with respect to epilepsy, some of these non-linear models have been shown to produce abrupt transitions to rhythmic oscillations, reminiscent of the epileptic discharge (Freeman, 1987).

The behavior of L over the preictal, ictal and postictal periods indicates that the system enters a less chaotic state after several transient decreases in chaoticity in the

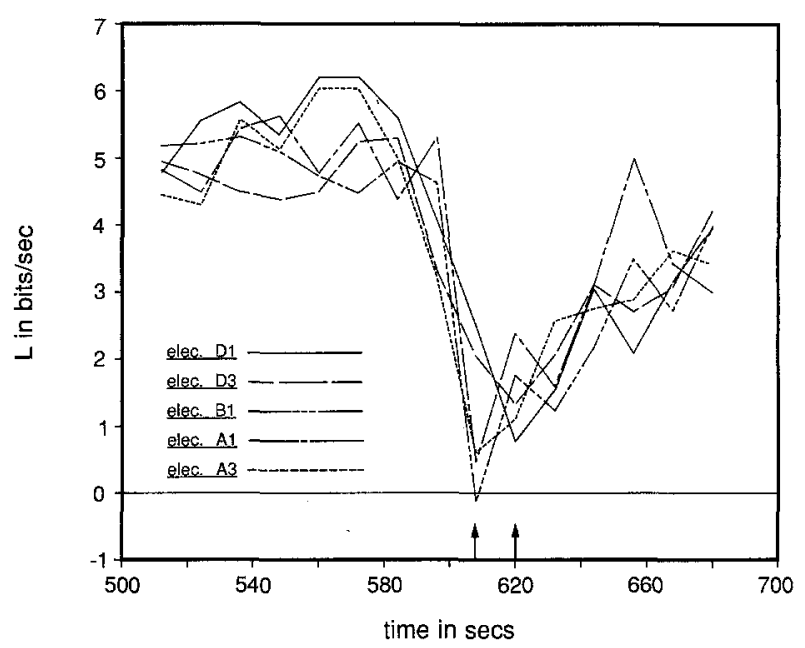

Figure 10 Transition from the preictal state to the ictal one: $L$ vs. time for electrodes $B 1, A 1, A 3$ overlying the ictal focus $\left(p=7, \tau=14 \mathrm{msec}, T=12 \mathrm{sec}, \Delta t=100 \mathrm{msec}, \partial_{\text {init }}=0.1 \mathrm{rad}\right.$, IMAX $=84 \mathrm{msec} b=0.05 . c=0.1$ ) and for electrodes DI and D3 outside the ictal focus.

preictal period. The attractor in the ictal state remains chaotic, although much less so than the preictal state. Several routes to chaos have been proposed (Grebogi et al. 1983; Martin et al. 1986). In the case of epilepsy the reverse route is to be considered, that is from chaos to order. Open questions about the seizure itself as a compensating mechanism (Rapp et al. 1986) and how it relates to the spiking process observed in the data still exist. We feel that an unambiguous answer to the above questions will be given only when the route of the system from the chaotic preictal state to the chaotic attractor state is thoroughly investigated.

The approach employed in our studies appears to be useful for identifying foci of interictal spike generation, for detecting and localizing ictal onset and for studying the spatial spread of ictal discharges. This approach could also provide an objective and quantitative method, based on the values of $L$ and $L^{*}$, for detecting segments of ECoG containing spikes and ictal discharges and for verifying spike and seizure localization. Application of these procedures to long-term monitoring in epilepsy appears to be feasible and promises to be useful. Further clinical studies will be required to determine the specificity and sensitivity of the methods. It remains to be seen whether or not the methods we have employed for analysis of the ECoG will be useful for analysis of EEG signals recorded form the scalp. The problems of muscle and movement artifacts encountered in scalp recordings of the EEG are not encountered in the ECoG.

A question that must be addressed is the time resolu- 

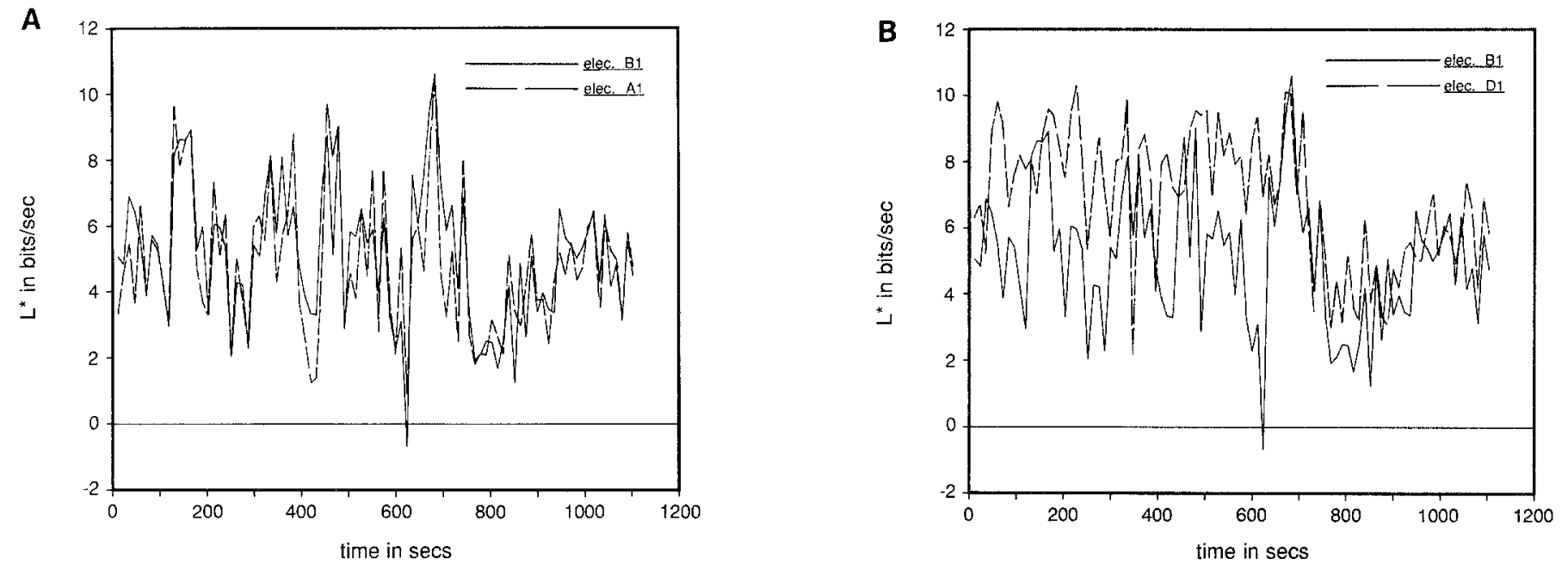

Figure $11 \mathrm{~L}^{*}$ vs.time for (a) electrodes B I, A1, (b) electrodes B 1, D 1.

tion of the current method for the estimation of $L$. The EcoG is a highly nonstationary signal. However, estimation of $L$ requires a large number of voltage values for a faithful reconstruction of the phase space and the estimates of $\mathrm{L}$ to converge. (In our current studies, each value of $\mathrm{L}$ was estimated from a $\mathrm{T}=12$ second epoch of the signal-see Figure 19 for the effect of the duration $T$ of the segment on L). There are really two questions of nonstationarity involved in the procedure. First within the segment under analysis, and secondly between segments (the second nonstationarity is not a serious theoretical problem since one can always use overlapping segments to follow more closely the change of $\mathrm{L}$ over time, which is more costly though). The first nonstationarity raises the question of how accurate is the estimated value of $L$ over a long segment. Problems of this nature occur especially when Wolf's algorithm for $\mathrm{L}$ is used without the proper modification (Wolf et al. 1986). We managed to take this into consideration by using the concept of local $X_{i, \max }(t)$ (Appendix B - formula B2) for every point $i$ of the fiducial trajectory in the phase space (results from signals of known models, for comparison reasons, with and without inserted nonstationarities are given in Appendix C).

The algorithm for the estimation of $\mathrm{L}$ is computer intensive in its present form. It takes about one minute CPU time on a mainframe IBM 3090-600E/VM computer for one estimate of $\mathrm{L}$ of 12 seconds of real time data embedded in a $p=7$ phase space. Improvements in the searching procedure and other parts of the algorithm can be made without a considerable trade off in the accuracy. We feel that dedicated work stations with custom digital signal processing hardware can accomplish this task in real time.

\section{Appendix A}

\section{Concepts of Phase Space, Attractor, Lyapunov Exponents, Chaos}

A dynamical system consists of two parts: the state (the essential information about the system at a time point) and a dynamic (the rule that describes how the state evolves with time).

A phase space is an abstract construct whose coordinates are the components of the state. The usefulness of the phase space picture lies in the ability to represent the state in geometric form. The temporal evolution of the system is visualized in the phase space as an orbit.

Dissipative systems (e.g. systems with losses of energy due to friction or viscosity) are characterized by the convergence of the orbits towards a smaller region of the phase space of lower dimension, which is called attractor. A system may have several attractors and different initial 
conditions (set of points in the phase space) may evolve to each of these attractors. Each set of these points constitute the basin of attraction for the corresponding attractor. If the attractor exhibits, under magnification, self-similar structure on all of the smaller scales or selfaffine behavior (structures on different scales are related by linear transformations) then it is a fractal (thin fractal if its dimension is a non integer, fat fractal if its dimension is an integer). An attractor that is a fractal is called strange. A chaotic attractor is an attractor in which two orbits with nearby initial conditions diverge exponentially fast, so they stay close together only for a short time $\Delta t$ (stretching process). Since they belong to the attractor and the attractor is of finite size, they have to come back into it as time evolves (folding process). The result is a layered structure (not always a fractal). Hence chaotic attractors are not in general strange (Jakobson 1981 and Li et al. 1978). Strange attractors may also exist that are not chaotic (Grebogi et al. 1984, Romeiras et al. 1987).

If the system is conservative (no dissipation of energy), it is always located on a surface of constant energy in the phase space. This system might be chaotic or nonchaotic (Eckmann et al. 1985).

A measure that quantifies the observed chaoticity is the average largest Lyapunov exponent. This measure is closely related to the average rate at which information is produced (Mayer-Kress 1986). It is defined as the average of $\mathrm{L}_{\mathrm{ij}}$;

$$
L=\frac{1}{N_{a}} \stackrel{\Sigma}{a} L_{i j}
$$

where $\mathrm{L}_{\mathrm{ij}}$ are the partial Lyapunov exponents, estimated from the evolution of the adjacent points $X_{i}, X_{i}$ in the phase space, defined by

$$
L_{i j}=\frac{1}{\Delta t} \cdot \log _{2} \frac{\left|X_{i}(t+\Delta t)-X_{j}\left(t^{\prime}+\Delta t\right)\right|}{\left|X_{i}(t)-X_{j}\left(t^{\prime}\right)\right|}
$$

$\mathrm{N}_{\alpha}$ is the number of the partial Lyapunov exponents estimated in the segment of duration $T$ under consideration ( $\left.T=N^{*} D t=N_{\alpha}{ }^{*} \Delta t\right) ; X_{j}$ are points in the phase space adjacent to each $X_{j} ; \Delta t$ is the evolution time in sec, Dt the sampling period, and $L$ in bits/sec (see Figure 12). The convergence of $L$ in (A1) with $N_{\alpha}$ is shown in Figure 13. The estimation of $\mathrm{L}$ is also discussed in Wolf et al. 1985. If the phase space is of p dimensions we can theoretically estimate up to $p$ Lyapunov exponents (Lyapunov spectrum). To estimate the second largest Lyapunov exponent L' we follow the expansion or the contraction of an area element in the phase space (this gives an estimate of $L+L^{\prime}$ ). For the third exponent $L^{\prime \prime}$ we measure the expansion or the contraction of volume elements and so on. If an attractor is a stable fixed point the spectrum of the Lyapunov exponents are the real parts of the eigenvalues of the linearized problem. For a chaotic attractor, there is no such simple correspondence.

\section{Appendix B}

\section{Comments on the Estimation of $L$ for $E C O G$}

The definition of $\mathrm{L}_{\mathrm{ij}}$ indicates that several parameters enter the estimation procedure. These are the evolution time $\Delta t$, the embedding dimension $p$, the time lag $\tau$ for the construction of the phase space, and finally the necessary parameters for the selection of $X_{i}(t)$ and $X_{j}\left(t^{\prime}\right)$ vectors in the phase space (Figure 12).

The steps to be followed in the estimation of $L$ are:

Step 1: Select $p$ such that the dimension D of the epileptic attractor in the ictal state is clearly defined. This is so if $\mathrm{p}>6$ (Iasemidis et al. 1988b; Rapp et al. 1986; Babloyantz et al. 1986). We used $p=7$ for all the L's plots. The variation of $L$ with $p$ in the preictal and ictal state is shown in Figure 14.

Step 2: Select $\tau$ such that (p-1) $\tau$ is approximately equal to the minimum period of any prominent sine wave component present in the data. After frequency analysis we found that the maximum dominant frequency in the seizure is about $11 \mathrm{~Hz}$ (Zaveri et al. 1988), so (p-1) $\tau \cong$ $90 \mathrm{msec}$. With $\mathrm{p}=7$ the value for $\tau$ is about $15 \mathrm{msec}$. Alternative procedures for estimation of $\tau$ are based on the first zero of the autocorrelation or the time constant (Rapp et al. 1988); in our data it was very difficult to decide on the basis of these criteria.

Step 3: Follow the evolution of $X_{i}(t)$ for $t=t_{0}, t_{0}+\Delta t$, $t_{0}+2^{*} \Delta t, \ldots$ with $0<t_{0}<\Delta t$. This is the fiducial orbit. For different departure values $t_{0}$, we have different fiducial orbits. For each one, a final $\mathrm{L}$ value is produced. The mean and standard deviation of these L's are given in Figure 15 as an uncertainty of the $L$ measures with respect to $t_{0}$.

Select $X_{j}\left(t^{\prime}\right)$ on the basis of how close in magnitude to $X_{i}(t)$ should be and on the basis of its angle deviation $\partial$ where 


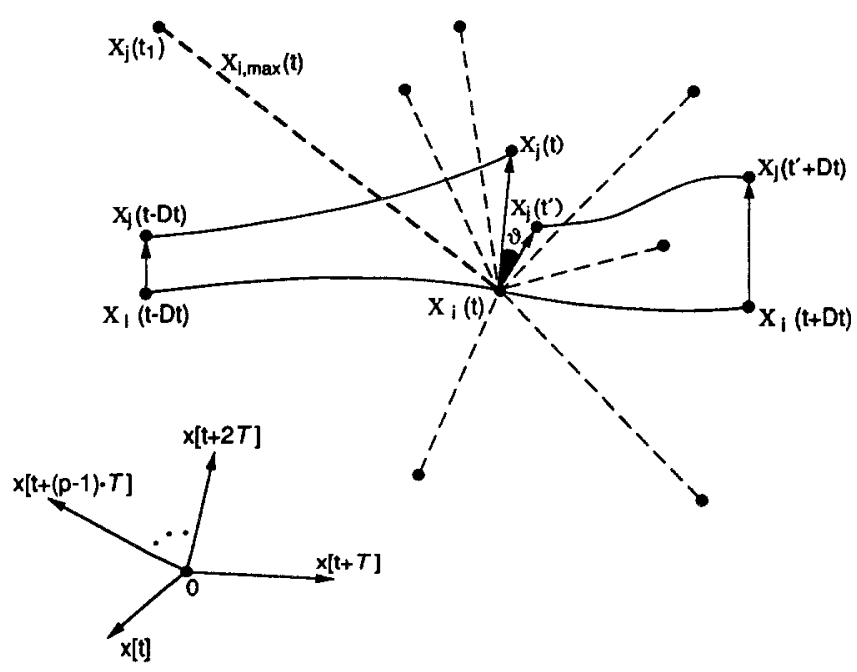

Figure 12 Diagram of the evolution and replacement procedure used to estimate the Lyapunov exponent $L$ for one segment of data.

$$
\partial(t)=\left\langle\left[X_{i}(t)-X_{j}(t)\right],\left[X_{i}(t)-X_{j}\left(t^{\prime}\right)\right]\right\rangle
$$

so that the direction of the flow in the phase space to be preserved.

Accurate construction (more computationally expensive though) requires small angle deviations $\partial$. We used initial minimum angle deviations of $\partial_{\text {init }}=0.1$ radians or 0.2 radians, doubling its value in case of not finding a replacement point. Results for different $\partial_{\text {init }}$ are given in Figure 16. The convergence of $L$ for $\partial_{\text {init }} \rightarrow 0.1$ radians is verified.

In the estimation of $L$ only ratios of vector differences take part so any DC present or the scaling of the data in the segment of interest do not influence the final result (as far as the pertinent parameters involved in the computing process are not given absolute values but relative ones to the scale of the data). The selection of $X_{j}\left(t^{\prime}\right)$ on the basis of its distance from $X_{i}(t)$ is a critical step. By using the maximum vector distance $X_{\max }=\max \left|X_{i}-X_{j}\right|$, with $j$ not equal to $i$ for all $i$ 's, as a bound for this distance (as in Wolf et al. 1985), the effect of spikes in the ECoG obscures the structure of the attractor. For spatial closeness, the solution is to choose $X_{j}\left(t^{\prime}\right)$ such that:

$b^{\prime} X_{i, \max }(t)<\left|X_{i}(t)-X_{j}\left(t^{\prime}\right)\right|<c \cdot X_{i}, \max (t)$

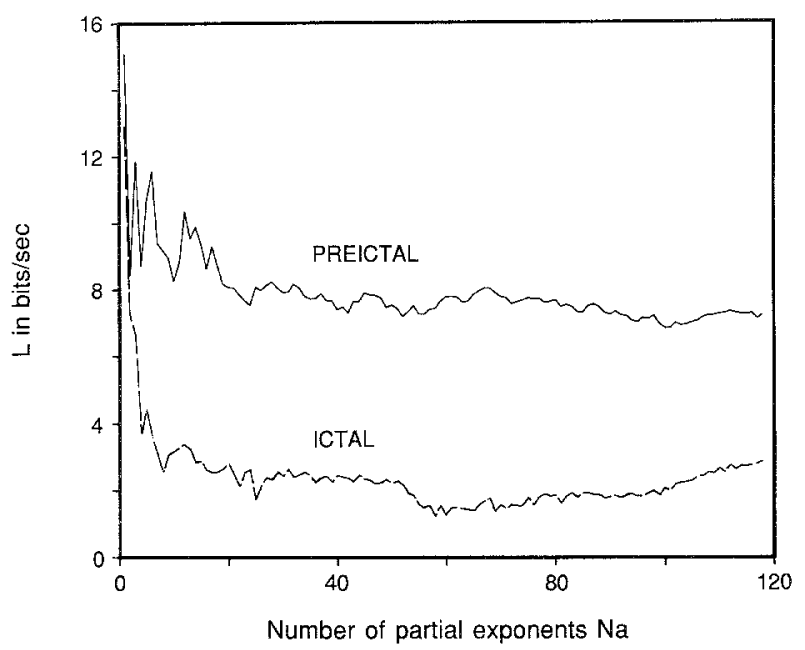

Figure 13 L vs. Na for preictal and ictal data segments (parameters as in figure 10).

where $X_{i, \max }(t)$ is the maximum distance from $X_{i}(t)$ of points in the phase space that are close in time to the $X_{i}(t)$, that is:

$$
\begin{gathered}
X_{i, \max }(t)=\max \left|X_{i}(t)-X_{j}\left(t_{1}\right)\right| \text { for } \\
I D I S T_{1}<\left|t-t_{1}\right|<I M A X
\end{gathered}
$$

By this estimation of $X_{i, \max }(t)$ at every point $X_{i}$ in the phase space and within the time window of (B2), using IDIST $_{1} \cong \tau=14 \mathrm{msec}$ the minimum acceptable distance, and IMAX $\cong(p-1) \cdot \tau=84 \mathrm{msec}$ we try to compensate for any nonstationarites present within the segment (state dependent estimation of $X_{i}$, max - see Figure 17 for the change of $L$ with IMAX).

Also, $\mathrm{c}$ changes from 0.1 to 0.5 by step 0.1 in case no replacement vector is found.

Parameter $b$ (must be smaller than $c$ ) was selected equal to 0.05 to take care of the noise level possibly present (see Figure 18 for dependence of $L$ on $b$ ).

In addition, the replacement point $X_{j}\left(t^{\prime}\right)$ should not be too close in time to $X_{i}(t)$ so that they do not belong to the same state; this means that

$$
\left|t^{\prime}-t\right|>I D I S T_{2}
$$

and $\operatorname{IDIST}_{2} \cong(\mathrm{p}-1) \cdot \mathrm{t}$ is the minimum reasonable value according to the above argument. 


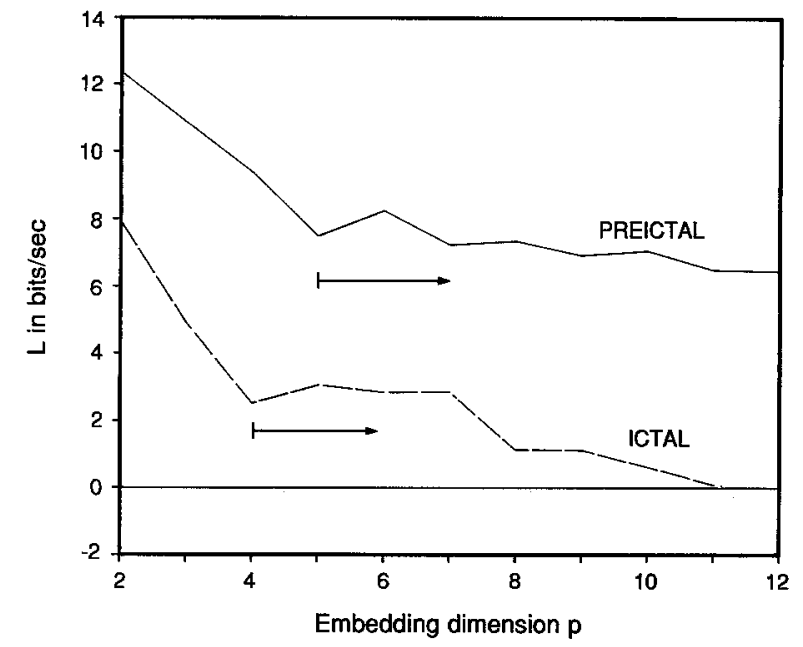

Figure $14 \mathrm{~L}$ vs. $p$ for one preictal and ictal segment (rest of parameters as in figure 10).

Step 4: The evolution time $\Delta t$ is highly dependent on the particular problem at hand. It is related to the folding process that takes place inside the attractor. Its value was calculated on the basis of convergence of $L$ for different values of $\Delta \mathrm{t}$ (Figure 19). The value $\Delta \mathrm{t}=80 \mathrm{msec}$ was selected.

Step 5: The time duration $\mathrm{T}$ of the segment for estimation of $L$ was $12 \mathrm{sec}(\mathrm{N}=6,000$ points). The heuristic value is $10^{\mathrm{D}}-30^{\mathrm{D}}$ where $\mathrm{D}$ is the dimension of the attractor (Wolf et al. 1985). Here $D$ is between 2 and 3 for the ictal case (Iasemidis et al. 1988b). So 1,000 $<\mathrm{N}<27,000$. The behavior of $L$ with $N$ is given in Figure 20.

\section{Appendix C}

Estimation of $\mathrm{L}$ for standardized examples from the literature

1. Limit cycle attractor $(D=1)$

Using the signal in Figure 2a, which is generated by

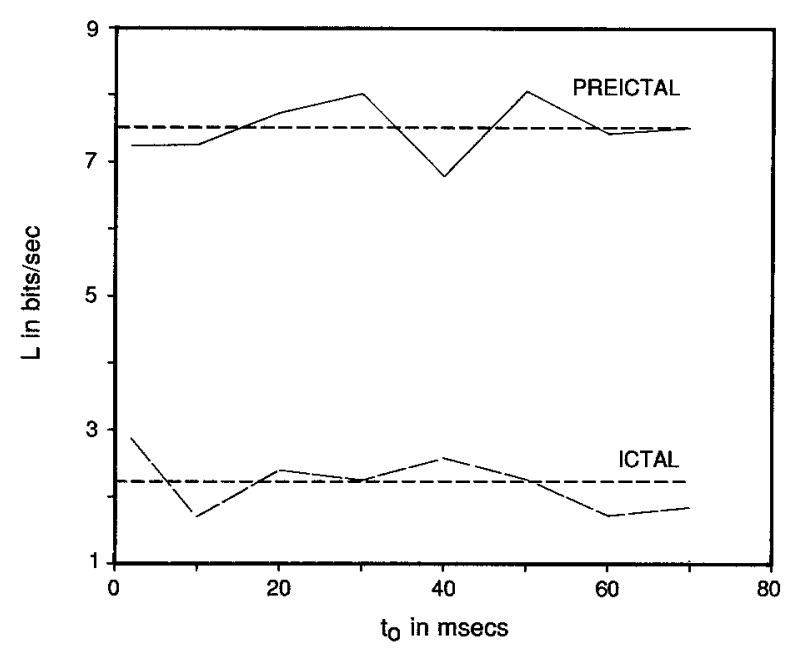

Figure $15 \mathrm{~L}$ vs. to for one preictal $(L=7.50 \pm 0.43 \mathrm{bits} / \mathrm{sec})$ and ictal $(L=2.21 \pm 0.42 \mathrm{bits} / \mathrm{sec}$ ) segment (rest of parameters as in figure 10).

$x(n)=\sin \left(\frac{2 \pi}{N_{0}} \cdot n \cdot 1\right)+\sin \left(\frac{2 \pi}{N_{0}} \cdot n \cdot 5\right)$ for $n=0, \ldots, N-1$ where $\mathrm{N}=2,000$ points and $\mathrm{N}_{0}=100$ points, the estimated $\mathrm{L}=0.00164$ bits/iteration with $\mathrm{p}=3, \tau=10$ points, $\Delta \mathrm{t}=100$ points, $\mathrm{IMAX}=100$ points, $\partial_{\text {init }}=0.1 \mathrm{rad}$. The theoretical value of $L$ here is 0 bits/iteration. The closest $L$ to zero we found was with $\mathrm{p}=6, \tau=20$ points $\left[(\mathrm{p}-1) \cdot \tau=\mathrm{N}_{0}\right], \Delta \mathrm{t}=40$ points, IMAX $=100$ points, $\partial_{\text {init }}=0.1 \mathrm{rad}, \mathrm{N}=6,000$ points and its value was $L=0.0004563$ bits/iteration.

\section{Torus attractor $(\mathrm{D}=2)$} by

We consider the signal depicted in Figure 4a generated $x(n)=\sin \left(\frac{2 \pi}{N_{0}} \cdot n^{\prime} 1\right)+\sin \left(\frac{2 \pi}{N_{0}} \cdot n^{\prime} \sqrt{2}\right)$ for $n=0, \ldots N-1$

where $\mathrm{N}=6,000$ points and $\mathrm{N}_{0}=100$ points. The estimation of $L$ with: $p=5$ (since $D=2, p=2 * D+1$ ), $\tau=25$ points [so $(\mathrm{p}-1)^{*} \tau=\mathrm{N}_{0} \mathrm{l}, \Delta \mathrm{t}=40$ points, $\mathrm{IMAX}=6,000$ points, $\partial_{\text {init }}=0.1$ rad, gives $\mathrm{L}=0.0027$ bits/iteration. The theoretical $\mathrm{L}$ value of this signal is 0 bits/iteration.

3. Henon attractor $(D=1.26)$ 


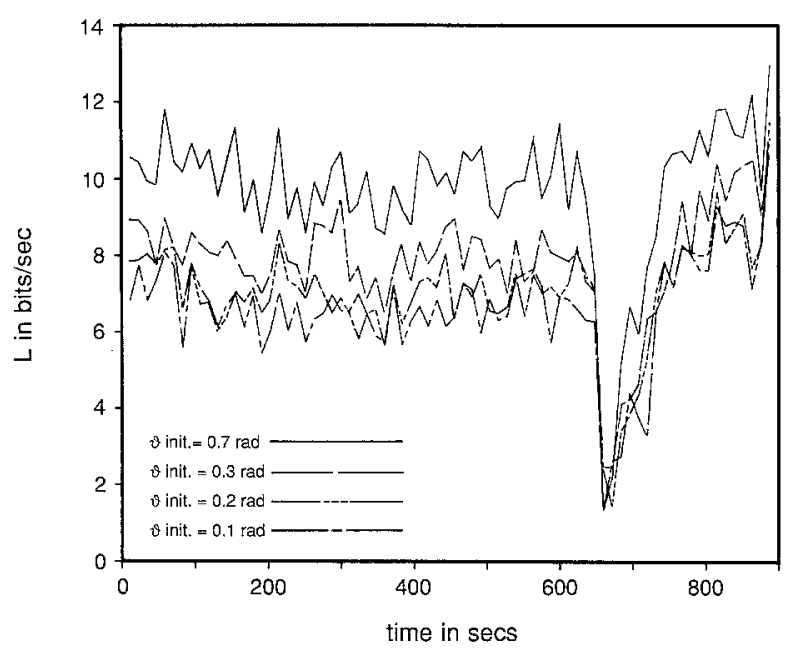

Figure $16 \mathrm{~L}$ vs. time for electrode $\mathrm{B} 1$ with: $(a) \partial_{\text {tnit }}=0.1 \mathrm{rad}$. (b) $\partial_{\text {init }}=0.2 \mathrm{rad}$, (c) $\partial_{\text {in } 1}=0.3$ rad (rest of parameters as in figure 10), (d) $\partial_{\text {init }}=0.7 \mathrm{rad}$.

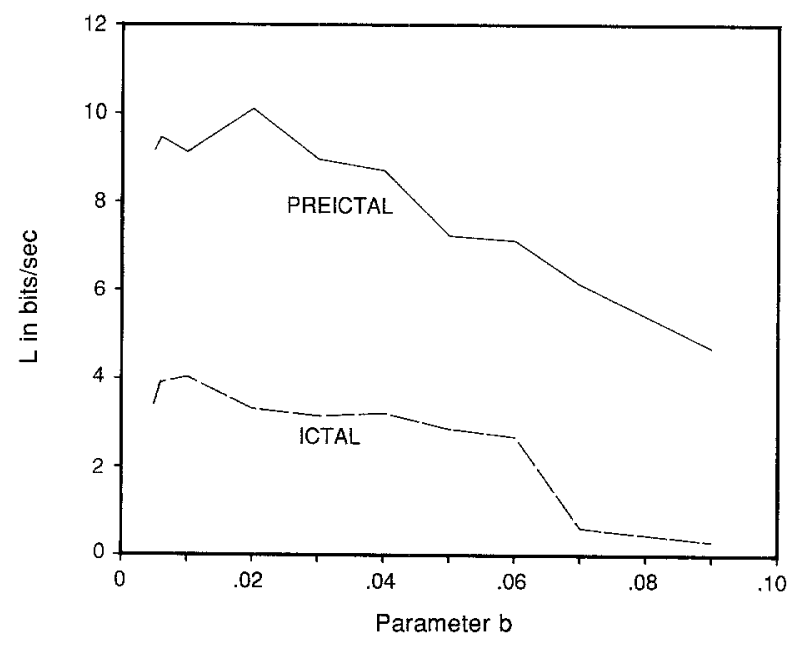

Figure $18 \mathrm{~L}$ vs. $b$; (rest of parameters as in figure 10).

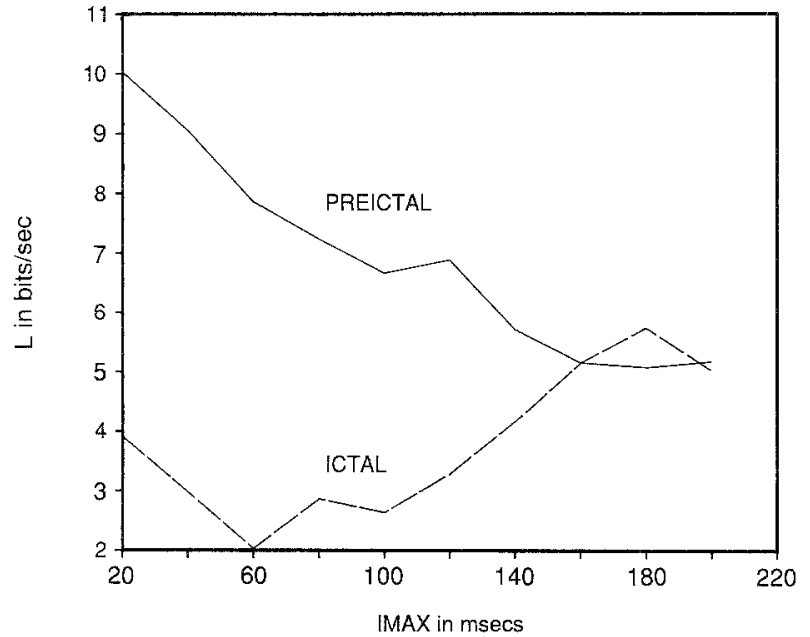

Figure $17 \mathrm{~L}$ vs. IMAX for the preictal and ictal segments (rest of parameters as in figure 10).

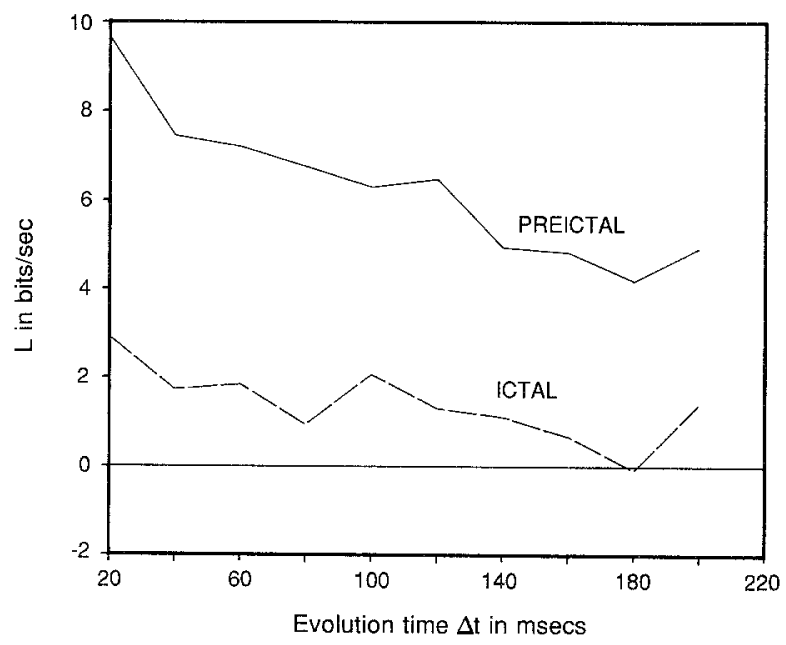

Figure $19 \mathrm{~L}$ vs. evolution time $\Delta t$ of one preictal and one ictal $12 \mathrm{sec}$ data segment from electrode 11 ( $\partial$ init $=0.2 \mathrm{rad}$, rest of parameters as in figure 10). 


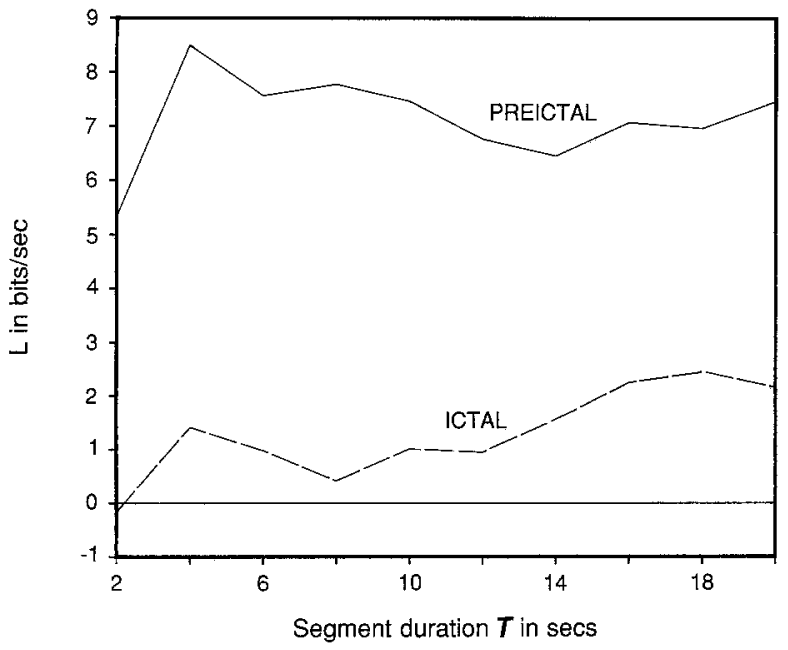

Figure $\mathbf{2 0} \mathrm{L}$ for different durations $\mathrm{T}$ of preictal and one ictal data segment (rest of parameters as in figure 10).

The data were produced by the following set of the difference equations:

$$
\begin{gathered}
x(n+1)=1-c \cdot x^{2}(n)+\psi(n) \\
\psi(n+1)=b \cdot x(n)
\end{gathered}
$$

with $c=1.4, b=0.3, x(0)=0.631, \psi(0)=0.189$ and $n=0, \ldots, N-1$. We select $\mathrm{N}=2,000, \mathrm{p}=2, \tau=1$ point (infinite frequencies present), $\Delta \mathrm{t}=1$ point, $\mathrm{IMAX}=2,000$ points, $\partial_{\text {init }}=0.1 \mathrm{rad}$. The estimated value of $\mathrm{L}$ was 0.577 bits/iteration. The theoretical value is 0.6 bits/iteration (Wolf et al. 1985).

\section{Henon attractor with one transient}

The signal used is $z(n)=x(n)+w(n)$ where $x(n)$ is produced from the previous Henon system and $w(n)$ a set of six consecutive impulses with amplitude four times the maximum amplitude of $x(n)$, inserted in the middle of the $x(n)$ record. Using the same parameters as in (3) we find $L=0.420$ bits/iteration. Now selecting IMAX $=100$ points, to reduce the effect of the $w(n)$ in the estimation of $\mathrm{L}$, we find $\mathrm{L}=0.556$ bits/iteration, which was the closest value to the theoretical $L=0.6$ bits/iteration of the pure Henon attractor.

\section{Simple quadratic map}

It is produced by the simple nonlinear difference equation $x(n+1)=a-x^{2}(n)$. With $a=2$ we are in the chaotic regime. Using $x(0)=0.1$ for $n=0, \ldots, N-1$, with $N=2,000$ points, $p=1, \tau=1$ point, $\Delta t=1$ point, $\partial_{\text {init }}=0.1 \mathrm{rad}$, IMAX $=2,000$ points, the estimated $L=1.097$ bits $/$ iteration. The theoretical value of $L$ is $\ln 2 /$ iteration or $\log _{2} 2=1$ in bits/iteration (Grassberger, 1985).

\section{References}

Babloyantz, A. and Destexhe, A. Low dimensional chaos in an instance of epilepsy. Proc. Natl. Acad. Sci. USA, 1986, 83:3513-3517.

Babloyantz, A. and Salazar, J.M. Evidence of chaotic dynamics of brain activity during the sleep cycle. Phys. Let., 1985, 111A:152-156.

Campbell, D.K. Nonlinear Science-From paradigms to practicalities. Los Alamos Science, Special Issue, 1985, 15:218262.

Eckmann, J. P., and Ruelle D. Ergodic theory of chaos and strange attractors. Rev. of Mod. Phys., 1985, 57:617-656.

Freeman, W.J. Simulation of chaotic EEG patterns with a dynamic model of the olfactory system. Biol. Cybernetics, 1987, 56:139-150.

Glass, L., Mackey, M.C. From Clocks to Chaos: The Rhythms of Life. Princeton University Press, 1988.

Gotman, J., Ives, J.R., Gloor, P. (Eds). Long-term monitoring in epilepsy. Electroenceph. and Clin. Neurophys., 1985, 35(suppl):93-201.

Grassberger, P. Information flow and maximum entropy measures for 1-D maps. Physica D, 1985, 14:365-373.

Grassberger, P. and Procaccia, I. Measuring the strangeness of strange attractors. Physica D, 1983, 9:189-208.

Grebogi, C., Ott, E., Pelikan, S., Yorke, J.A. Strange attractors that are not chaotic. Physica D, 1984, 13:261-268.

Grebogi, C., Ott, E., Yorke, J.A. Crises, sudden changes in chaotic attractors and transient chaos. Physica D, 1983, 7:181-200.

Holzfuss, J., Mayer-Kress, G. An approach to error estimation in the application of dimension algorithms. In: G. MayerKress (Ed.), Dimensions and Entropies in Chaotic SystemsQuantification of Complex Behavior. Springer-Verlag, Springer Series in Synergetics, Berlin, 1986, 32:114-122.

Iasemidis, L.D., Zaveri, H.P., Sackellares, J.C. and Williams, W.J. Modelling of ECoG in temporal lobe epilepsy. 25th Ann. Rocky Mountain Bioeng. Symposium, 1988(a), 24:187193.

Iasemidis, L.D., Zaveri, H.P., Sackellares, J.C. and Williams, W.J. Phase space analysis of EEG in temporal lobe epilepsy. IEEE Eng. in Medicine and Biology, 10th Ann. Int. Conf., 1988(b):1201-1203.

Jakobson, M.V. Absolutely continuous invariant measures for one parameter families of one dimensional maps. Comm. Math. Phys., 1981, 81:39-88.

Layne, S., Mayer-Kress, G., Holzfuss, J. Problems associated with the dimensional analysis of electroencephalogram data. In: G. Mayer-Kress (Ed.), Dimensions and Entropies in Chaotic Systems-Quantification of Complex Behavior. Springer-Verlag, Springer Series in Synergetics, Berlin, 1986, 32:246-256. 
Li, T.V., Yorke, J.A. Ergodic tansformations from an interval into itself. Trans. Am. Math. Soc., 1978, 235:183-192.

Martin, S., Martienssen, W. Transition from quasiperiodicity into chaos in the periodically driven conductivity of BSN crystals. In: G. Mayer-Kress (Ed.), Dimensions and Entropies in Chaotic Systems-Quantification of Complex Behavior. Springer-Verlag, Springer Series in Synergetics, Berlin, 1986, 32:191-197.

Mayer-Kress, G., Holzfuss, J. Analysis of the human electroencephalogram with methods from nonlinear dynamics. In: L. Rensing, U. ander Heiden, M.C. Mackey (Eds.), Temporal Disorder in Human Oscillatory Systems, Springer Series in Synergetics, Springer-Verlag, Berlin, 1986, 36:57-68.

Ott, E. Strange attractors and chaotic motions of dynamical systems. Reviews of Mod. Phys., 1981, 53:655-671.

Packard, N.H., Crutchfield, J.P., Farmer, J.D. and Shaw R.S. Geometry from a time series. Phys. Rev. Let., 1980, 45:712716.

Rapp, P.E., Albano A.M., Mees A.I. Calculation of correlation dimensions from experimental data: Progress and problems. In: J.A.S. Velso, A.J. Mandell, M.F. Schlessinger (Eds.), Dynamic Patterns in Complex Systems. World Scientific, 1988:191-205.

Rapp, P.E., Zimmerman, I.D. Albano, A.M., deGuzman, G.C., Greenbaum, N.N. and Bashore, T.R. Experimental Studies of chaotic neural behavior: cellular activity and electroencephalographic signals. In: H.G. Othmer (Ed.), Nonlinear Oscillations in Biology and Chemistry. Springer-Verlag, Berlin, 1986:175-805.

Romeiras, F.J., Bondeson, A., Ott, E., Antonsen, T.M. Jr., Grebogi, C. Quasiperiodically forced dynamical systems with strange nonchaotic attractors. Physica D, 1987, 26:277294.

Swinney, H.L. Observations of order and chaos in nonlinear systems. Physica D, 1983, 7:3-15.

Takens, F. Detecting strange attractors in turbulence. In: D.A. Rand and L.S. Young (Eds.), Dynamical Systems and Turbulence. Lecture Notes in Mathematics. Springer-Verlag, Berlin, 1981:366-381.

Vastano, J.A., Kostelich, E.J. Comparison of algorithms for determining Lyapunov exponents from experimental data. In: G. Mayer-Kress, (Ed.), Dimensions and Entropies in Chaotic Systems-Quantification of Complex Behavior. Springer-Verlag, Springer Series in Synergetics, Berlin, 1986, 32:100-107.

Whitney, H. Differentiable Manifolds. Ann. Math., 1936, 37:645-680.

Wolf, A. Quantifying chaos with Lyapunov exponents. In: A.V. Holden (Ed.), Chaos. Princeton University Press, New Jersey, 1986:270-290.

Wolf, A., Swift, J.B., Swinney, H.L. and Vastano, J.A. Determining Lyapunov exponents from a time series. Physica D, 1985, 16:285-317.

Wolf, A., Vastano, J.A. Intermediate length scale effects in Lyapunov exponent estimation. In: G. Mayer-Kress (Ed.), Dimensions and Entropies in Chaotic Systems-Quantification of Complex Behavior. Springer-Verlag, Springer Series in Synergetics, Berlin, 1986, 32:94-99.

Zaveri, H.P., Iasemidis L.D., Williams, W.J., Sackellares, J.C. Multielectrode analysis of the electrocorticogram in temporal lobe epilepsy. IEEE Eng. in Medicine and Biology, 10th Annual International Conference, 1988:1198-1200. 